\title{
The benefits of Scanning Precession Electron Diffraction to access to the crystallographic phases and orientations of nanomaterials
}

\author{
Edgar F Rauch \\ University Grenoble Alpes - CNRS, Saint Martin d'Hères, France; \\ edgar.rauch@simap.grenoble-inp.fr
}

There is a renewal in diffraction experiments with a Transmission Electron Microscope promoted by their coupling with the scanning mode. In these approaches, 2D diffraction patterns are systematically acquired with fast cameras while the focused beam is moved point by point across a 2D field of view.

Depending on the probe size the resulting 4D datasets enables the reconstruction of 2D maps highlighting either structural entities at atomic scale (when the probe convergence angle is large) or phases, crystallographic orientation and/or local stress fields at the nanoscale (for convergence angles of few mrad). Only the latter case - that ends with spot patterns - will be considered in the present work. The identification of these patterns is substantially simplified when precession electron diffraction (PED) is used. Precession allows more reflections to be captured and their intensities to be less sensitive to dynamical effects.

A significant advance related to scanning precession electron diffraction (SPED) with respect to the classical diffraction experiments is that the full set of patterns are memorized and available for further analysis. A pioneering post-processing work is the automate recognition of phases and crystallographic orientations through template matching, i.e.: by comparing the experimental patterns with a set of simulated ones and selecting the best fit in the pattern library to deduce the local crystal characteristics. Another popular facility offered by the availability of the data is the construction of so-called Virtual Bright- or Dark-Field images (VBF/VDF). These images are formed by plotting, pixel by pixel, the intensity of a user-selected reflection. The interesting point is that a 4D dataset provides the access to all dark-field images, to their combinations and allows the reconstruction of annular dark-field images by summing the intensity of incoherently scattered electrons at the rim of the patterns.

The most challenging problem for transmission diffraction patterns is the frequent crystals overlapping in nanoscaled materials. The related patterns contain the diffracting spots of all superimposed crystals in the thin foil. This is usually considered as a drawback as it renders their analysis less straightforward. It can also be seen as an opportunity that allows the 3D nature of the superimposed grains to be analyzed, by contrast to techniques, like EBSD or Transmission Kikuchi Diffraction, that give access to a surface information solely. For example, Correlation Coefficient Maps (CCM), obtained by measuring the degree of similarity between successive patterns, highlight inner boundaries and, in some conditions give access to their angle of inclination.

There are several existing approaches to isolate the superimposed information: they make use of dark-field images, involve multivariate analyses or include image segmentation algorithms. The proposed solution consists in generating a collection of components that are recognized through template matching and then to construct template related VDF images. Each image gathers the intensities of all the reflections related to a given crystal and are processed in such a way that the resulting weighted intensity improves grain recognition. With this approach each component is related to a given phase and/or orientation and consequently has a physical meaning. Non dominant - or hidden - crystals are accessible with the procedure. A workflow towards 3D reconstructions at nanoscale with electron diffraction and tomographic approaches will be presented.

Keywords: Scanning Precession electron Diffraction ; Automated Crystal Orientation Mapping 